\title{
Ce que la médiatisation fait aux problèmes
} éducatifs

\section{Introduction}

Introduction: what media coverage does to educational issues

Introducción: los efectos de la mediatización sobre los problemas educativos

\section{Xavier Pons}

\section{OpenEdition}

\section{Journals}

\section{Édition électronique}

URL : http://journals.openedition.org/ries/3987

DOI : $10.4000 /$ ries.3987

ISSN : 2261-4265

\section{Éditeur}

Centre international d'études pédagogiques

\section{Édition imprimée}

Date de publication : 1 septembre 2014

Pagination : 23-30

ISBN : 978-2-85420-604-3

ISSN : 1254-4590

\section{Référence électronique}

Xavier Pons, "Ce que la médiatisation fait aux problèmes éducatifs", Revue internationale d'éducation de Sèvres [En ligne], 66 | septembre 2014, mis en ligne le 01 septembre 2014, consulté le 22 juin 2020 URL : http://journals.openedition.org/ries/3987 ; DOI : https://doi.org/10.4000/ries.3987 


\title{
L’école dans les médias
}

\author{
Introduction
}

\section{Ce que la médiatisation fait aux problèmes éducatifs}

\author{
Xavier Pons
}

Ce numéro aborde une série de questions simples, encore peu étudiées dans l'espace francophone : comment l'école est-elle évoquée dans les médias ${ }^{1}$ dans divers pays ? Quelles sont les différences entre le fonctionnement des institutions scolaires ( l'école réelle») et la façon dont on en parle dans les médias ( l'école médiatisée») ? Quels sont les enjeux cognitifs, politiques et professionnels de cette médiatisation, ses effets sur le débat public et ses implications pour les acteurs de l'éducation?

Pour y répondre, huit auteurs, journalistes et chercheurs en éducation, ont été invités, avec leur pleine liberté d'investigation, à traiter un ou plusieurs des points suivants :

- les caractéristiques de la production médiatique sur l'école (contraintes du travail journalistique, types de médias et de formats privilégiés, thèmes couverts, problématiques majeures ou minorées, profils, visions de l'école et prises de position des journalistes);

- les représentations de l'univers scolaire dans les médias (quelles images de l'école et de l'acte éducatif ? Quelle construction de sens ? Quels stéréotypes et idées reçues sur l'école?);

- les effets de la médiatisation sur les professionnels et les politiques de l'éducation (réorientation des problématiques et des pratiques éducatives? Mise en visibilité de problèmes ou de catégories spécifiques?) et les stratégies de médiatisation des problèmes éducatifs et de communication dans les médias (quelle mobilisation des médias par les acteurs? Quelles instrumentalisations politiques des médias? Poids et caractéristiques des communications politiques et institutionnelles?).

1. Les médias désignent ici les médias professionnels traditionnels ainsi que les nouveaux médias (médias numériques, réseaux sociaux, etc.). 


\section{LA MÉDIATISATION VUE PAR LA RECHERCHE INTERNATIONALE EN ÉDUCATION}

Ces questions et ces points d'entrée nous ont été inspirés par la littérature scientifique internationale sur le sujet, beaucoup plus développée dans l'univers anglophone ${ }^{2}$. Cette littérature met en évidence trois grandes caractéristiques du processus de médiatisation et divers effets de ce dernier sur le débat public, qui souvent vont à rebours de l'image spontanée qu'on peut en avoir.

La première, qui ressort des travaux lancés dans les années 1980 et qu'on retrouve dans plusieurs recherches actuelles, est que la médiatisation, qui apparaît souvent aujourd'hui comme une arène "bruyante » dans laquelle s'opposent différents points de vue, contribue en fait à stabiliser un consensus sur divers sujets (Lingard \& Rawolle, 2004), notamment parce qu'elle tend à conforter des présupposés culturels tacites, qui, par exemple, excluent le plus souvent la prise en compte dans les discours et les analyses des préoccupations des dominés (minorités culturelles, pauvres, exclus).

Contrairement à l'idée répandue, y compris parfois parmi les journalistes, selon laquelle la médiatisation permettrait d'ouvrir le débat public au plus grand nombre et contribuerait ainsi à son extension et à son amplification, certains chercheurs, comme Cynthia I. Gerstl-Pepin (2007) ayant travaillé sur la couverture médiatique de l'éducation lors des élections présidentielles américaines, concluent au contraire à une réduction des enjeux traités dans le débat public à la suite de leur médiatisation, les médias constituant selon elle une sphère publique étroite (thin public sphere), un prisme de diffusion finalement beaucoup plus limité qu'on ne l'imagine spontanément. Cette réduction se manifeste de plusieurs manières. De nombreux travaux, montre-t-elle, mettent en évidence que la médiatisation conduit à une représentation négative dans la presse nationale des problèmes de politique éducative et plus globalement à une simplification abusive des enjeux, en particulier dans le cas du traitement des différents palmarès disponibles sur les établissements et les systèmes scolaires. On retrouve cette problématique en France dans le cas de Pisa (Pons, à paraître), au sujet du débat sur la «crise » de l'école (Balland, 2009) ou dans la façon dont la presse évoque certaines questions pédagogiques (Forestier, 2014). Cette réduction se traduit également par la primauté donnée à la parole des responsables politiques et aux réactions du "public » à leurs annonces qui ne permet pas d'analyser les problèmes de fond posés par un sujet, en particulier à la télévision. Ceci peut conduire dans certains cas à des situations où la construction d'un discours médiatique sur une politique pallie de fait l'absence d'une politique

\footnotetext{
2. Les éléments de synthèse de cette littérature qui suivent se fondent en particulier sur les contributions aux numéros spéciaux des Cahiers de la recherche sur l'éducation et les savoirs ( $\left.n^{\circ} 1-2002\right)$, du Journal of Education Policy (vol. 19, $\mathrm{n}^{\circ}$ 3, 2004), du Canadian Journal of Education (vol. 29, $\mathrm{n}^{\circ}$ 1, 2006), du Peabody Journal of Education (vol. 82, $\mathrm{n}^{\circ}{ }_{1,2007}$ ) et de la revue Policy Futures in Education (vol. 5. $\mathrm{n}^{\circ}$ 1, 2007).
} 
plus substantielle (c'est notamment le cas de certaines "politiques» de lutte contre la violence à l'école, en France et ailleurs). L'approche du champ médiatique proposée par Bourdieu, souvent reprise au niveau international (Lingard \& Rawolle, 2004), fournit plusieurs éléments d'explication de ce processus de réduction. Selon lui, ce champ se caractérise par une circulation circulaire de l'information résultant de la forte compétition entre les médias, une amnésie permanente et structurelle selon laquelle, pour plusieurs raisons, les acteurs n'auraient qu'une faible mémoire des enjeux et une forte proximité avec le champ politique, qu'il influence et réciproquement.

Troisième caractéristique, la médiatisation est fondamentalement une mise en récit, voire une mise en intrigue, susceptible de redéfinir les causalités à l'œuvre dans le problème évoqué. Souvent cela donne lieu à une logique de présentation des faits particulière, celle de la contestation et de la mise en opposition des acteurs et de leurs discours. C'est particulièrement visible pour les palmarès en Angleterre, mais aussi en France par exemple avec le débat entre "pédagogues» et " (néo-)républicains », deux catégories d'analyse d'origine journalistique (Vergnioux, 2013 ; Forestier, 2014). La médiatisation peut alors devenir un élément constitutif d'une politique éducative, en particulier dans un contexte où un gouvernement accorde une place centrale à la communication sur ses actions. C'est ce que montrent Sharon Gewirtz, Marny Dickson et Sally Power (2004) dans le cas de la politique travailliste anglaise d'éducation prioritaire, où une faute de communication politique, et sa très mauvaise réception dans le débat public après avoir été relayée par la presse, a amené le gouvernement à considérablement infléchir son discours et ses actions en matière d'association de partenaires privés, pour mieux mettre en avant le dynamisme et les initiatives des écoles dans la construction de partenariats.

\section{LES CONTRIBUTIONS DU NUMÉRO}

Comme le lecteur pourra le découvrir, ce numéro 66 de la Revue internationale d'éducation de Sèvres s'inscrit dans la continuité de ces réflexions - plusieurs auteurs contribuent d'ailleurs activement, dans leur pays et au niveau international, à leur diffusion - mais il ne s'y inscrit que partiellement, dans la mesure où il met aussi en lumière d'autres aspects du processus de médiatisation de l'école et de ses effets.

\section{Les multiples formes de réduction des enjeux}

On retrouve ainsi, dans plusieurs articles, le processus de réduction des enjeux auquel donne lieu la médiatisation. Celui-ci se traduit, dans certains cas, par une focalisation très forte sur une controverse simple qui tend à occuper une place importante, voire hégémonique dans le débat médiatique. L'article de 
Peter Wilby, journaliste anglais en éducation expérimenté et lui-même auteur d'articles dans des revues scientifiques, montre bien le pouvoir de cadrage du débat médiatique rempli par l'opposition entre progressistes et traditionnalistes à partir des années 1980, dans un contexte de politisation et de centralisation du débat sur l'éducation en Angleterre. Cet article fait en cela directement écho à celui de Yann Forestier, qui a récemment soutenu une thèse conséquente sur la couverture des questions pédagogiques dans la presse écrite française entre 1959 et 2008. L'historien montre que la querelle médiatique entre " républicains » et "pédagogues » est privilégiée dans les médias français moins pour sa pertinence propre que pour sa conformité aux normes en pleine évolution du métier de journaliste ainsi que pour sa capacité à relayer les préoccupations spécifiques d'un groupe social étroitement lié au monde de la presse dite de référence. Chacun à leur manière, et à partir de contextes différents, ils posent la question de la place problématique accordée aujourd'hui à une spécialité journalistique en éducation dans le traitement médiatique de ce domaine. La réduction des enjeux n'est-elle pas due aussi au privilège symbolique souvent donné, en matière d'analyse finale, aux journalistes issus des rubriques politiques ou à des éditorialistes qui entrent en général peu dans le détail des questions éducatives elles-mêmes ?

Ce phénomène de réduction est visible également dans l'importance prise, dans chaque pays, en matière de couverture médiatique, par les sujets hautement prévisibles du fait de leur grande régularité. Là encore, il est frappant de constater combien ces sujets " prêt-à-publier » que nous décrit Michelle Stack, professeure associée à l'Université de British Columbia, dans le cas du Canada (les palmarès ou les violences à l'école) peuvent être proches de ceux d'autres pays, comme les fameux «marronniers » qu'évoquent les journalistes français (la rentrée, le baccalauréat, telle agression d'enseignant...), et combien ils peuvent éclipser des problématiques de fond plus rarement abordées, souvent structurelles (comme la marchandisation et ses effets au Canada). Enfin, la réduction opère aussi au sein même de ces sujets prévisibles, comme le montrent Branka Cattonar et Eric Mangez, respectivement chargée de cours et professeur à l'Université catholique de Louvain, dans leur analyse de la couverture de Pisa dans la presse écrite en Belgique francophone (cette presse tendant à se focaliser de manière classique sur les résultats moyens, l'inefficacité et le manque d'équité du système scolaire belge).

Ce phénomène de réduction donne lieu parfois à une véritable dramatisation des enjeux, perceptible dans plusieurs articles. Branka Cattonar et Eric Mangez montrent ainsi que le « choc » Pisa est largement une construction médiatique en Belgique francophone. Peter Wilby revient sur le rôle des Black Papers en Angleterre dans la politisation de la couverture médiatique de l'éducation. En Australie, Sue Thomas, professeure associée à l'Université Griffith, montre même, dans le cas de la mise en ouvre d'un nouveau programme de tests des élèves, que cette dramatisation, substrat important d'un grand nombre 
de productions médiatiques, peut donner lieu à des discours contradictoires selon les contextes. Elle remarque ainsi que les discours dominants sont particulièrement critiques envers le programme lors de son lancement, du fait du stress supplémentaire causé aux élèves et à leur famille, puis qu'ils sont toujours aussi critiques ensuite, mais pour mieux pointer la responsabilité de l'institution et des parents dans les moindres résultats de leur enfant.

Cette recherche des responsables est également l'une des conclusions à laquelle arrive Moira O'Neil, chercheuse à l'Institut FrameWorks, dans son analyse d'un corpus important d'articles de presse, d'émissions télévisées diffusées à l'échelle nationale et de blogs à orientation politique sur les questions d'éducation aux États-Unis. L'auteure montre que trois imaginaires principaux se dégagent de ce corpus, trois façons dominantes de représenter l'école dans les médias (elle parle plus précisément de «cadres », frames) : le cadre consumériste, qui explique le fonctionnement du système éducatif à travers la métaphore de l'entreprise, le cadre de la «bulle » scolaire, qui évoque les questions d'apprentissage de manière exclusive et le cadre de la «crise », qui repose de manière régulière la question du niveau des élèves. Elle prouve, y compris statistiquement, que ces cadres sont envisagés séparément dans les médias et s'interpénètrent très peu, de sorte que les problèmes d'apprentissage, par exemple, sont rarement pensés en lien avec le fonctionnement du système scolaire et les politiques mises en œuvre pour les pallier, et réciproquement. Ce hiatus amène les journalistes, et plus globalement, à travers eux, l'opinion publique, à chercher les causes des problèmes observés dans les comportements individuels des acteurs (élèves, parents, enseignants) plutôt que dans des logiques de système.

\section{Trois autres pistes d'analyse}

Néanmoins, ce numéro ne saurait se réduire lui-même à une nouvelle illustration de ce phénomène de réduction, du fait de la richesse des cas étudiés pris individuellement d'abord, mais aussi parce que la prise en compte de ces derniers dans leur ensemble ouvre d'autres pistes d'analyse. Nous en distinguons trois. La première, et c'est l'un des apports d'une publication comme la Revue internationale d'éducation de Sèvres, est de montrer combien cette couverture médiatique peut varier en fonction des contextes. Ces derniers renvoient d'abord aux caractéristiques des systèmes scolaires, notamment leur structure. Puisqu'au Canada, les responsabilités en matière éducative sont essentiellement provinciales, nous dit Michelle Stack, la presse tend à ne couvrir au niveau national que des événements médiatiques significatifs à ce niveau, c'est-à-dire des manifestations d'ampleur, des faits divers choquants, des palmarès proposant des données comparatives... en clair ce que l'auteure appelle des « ready-made ». La situation anglaise décrite par Peter Wilby avant la centralisation et la politisation du débat éducatif en Angleterre est similaire, comme celle aux États-Unis avant le programme No Child Left Behind et en dehors des élections présidentielles. 
Ces contextes renvoient aussi aux propriétés morphologiques et aux dynamiques du débat public en éducation, qui dépendent elles-mêmes des contextes sociopolitiques. Branka Cattonar et Eric Mangez introduisent bien le fait qu'on ne peut comprendre le débat médiatique sur Pisa en Belgique francophone (son ampleur, la construction médiatique d'un " choc Pisa » et ses usages par la classe politique), sans tenir compte de l'élément de contexte primordial que constitue le modèle de la démocratie consociative ${ }^{3}$ et le principe de discrétion qui la gouverne. L'interview d'Abdou Salam Sall, président du comité des Assises nationales de l'éducation au Sénégal et ancien recteur de l'Université Cheikh Anta Diop (UCAD) de Dakar, nous met immédiatement aux prises avec un contexte sénégalais tout à fait différent, caractérisé par le faible développement d'une presse spécialisée en éducation et les nombreuses difficultés à surmonter (politiques, financières, professionnelles) pour espérer pouvoir parvenir à une couverture médiatique plus diversifiée et plus informée. Plus globalement, dans plusieurs articles (Angleterre, Australie, États-Unis, France, Sénégal), on observe que la politisation des sujets est souvent un préalable essentiel à leur médiatisation.

La deuxième piste d'analyse est que la médiatisation peut aussi permettre d'ouvrir certaines brèches dans le débat public et que la sphère publique étroite que constituent les médias ne conduit pas mécaniquement à une réduction des enjeux mais peut aussi contribuer dans certains cas à mettre l'accent sur de nouvelles problématiques. C'est particulièrement visible dans le cas de l'Allemagne, évoqué par Jeanne Rubner, journaliste allemande en éducation, auteure de plusieurs ouvrages sur le sujet et actuellement responsable de la politique éditoriale du service public audiovisuel de la Bavière en matière d'éducation. Ainsi la médiatisation de Pisa n'a pas seulement conduit à des transformations d'importance en Allemagne conformément à la métaphore du "choc Pisa », idée si communément admise dans le débat allemand qu'elle a pu donner lieu à une page sur Wikipedia ${ }^{4}$. Elle a aussi transformé le paysage médiatique allemand lui-même, les débats sur l'éducation pouvant désormais s'inscrire plus facilement dans un cadre national. En Belgique francophone, la médiatisation de Pisa a même joué un rôle de "tiers évaluateur» (expression que Branka Cattonar et Eric Mangez empruntent à Pierre Rosanvallon) mettant les élites de l'État sous une nouvelle forme de surveillance par des citoyens pouvant les interpeller, sur la base de cette nouvelle enquête. La médiatisation de l'éducation enfin peut

3. Une démocratie consociative, néologisme inventé par le politologue néerlandais Arendt Lipjhart combinant les termes « consensus » et " association », désigne un régime politique dans lequel le gouvernement recherche de manière prioritaire le consensus entre les nombreux groupes, minorités ou segments qui composent une société ou un pays, en général de petite taille. Pour l'auteur, quatre conditions doivent être réunies pour qu'une telle démocratie existe : des élections proportionnelles, des accords entre les différents composants de la société, un minimum d'autonomie de ces dernières et la possibilité donnée aux minorités de s'opposer au gouvernement et aux mesures qu'il mène.

4. http://de.wikipedia.org/wiki/PISA-Studien. 
même faire partie intégrante de tout un projet de transformation de la société. L'initiative « vacances citoyennes ", décrite par Abdou Salam Sall, le montre bien. Il y a, dans ce programme qui consiste à envoyer des étudiants dans les terroirs pour qu'ils y rendent des services à la population (déboisement, campagne sanitaire, alphabétisation, etc.), plus que des enjeux de formation. Il s'agit aussi, à travers une médiatisation que l'auteur souhaite plus technique et plus documentée, de donner une autre image de l'université dans les médias ; une université qui n'est pas qu'un héritage problématique de la colonisation et qui peut faire partie de la culture sénégalaise pour entrer ainsi dans un cercle de développement vertueux.

Enfin, la dernière piste d'analyse concerne un objet particulier, que nous croisons plus que régulièrement dans ce numéro, mais aussi dans les lectures qui ont permis de le préparer : le palmarès (ranking). On le voit notamment dans la bibliographie conçue par Bernadette Plumelle, responsable du centre de ressources et d'ingénierie documentaires du Centre international d'études pédagogiques (CIEP) : la médiatisation des tests, évaluations et palmarès a beaucoup retenu l'attention des chercheurs. Comment expliquer le succès médiatique des palmarès, dans plusieurs secteurs d'action publique d'ailleurs (universités, hôpitaux, etc.), au niveau national comme international (palmarès des grandes métropoles par exemple) ? Ce numéro propose plusieurs pistes explicatives, sans prétendre à l'exhaustivité. Le palmarès semble tout d'abord se fondre particulièrement bien dans le fonctionnement du champ médiatique. Ses promoteurs misant parfois explicitement sur le " coup médiatique ", il assume, voire vise, la circulation circulaire de l'information qu'évoque Bourdieu. Rarement contextualisé tout en produisant des éléments de comparaison dans le temps, il ne remet pas non plus fondamentalement en cause l'amnésie structurelle des médias, et contribue même peut-être à la masquer. Souvent conçu pour éclairer la décision, il permet une interpellation directe des responsables politiques amenés à réagir. Comme le précise Michelle Stack, il fournit des histoires rapidement mobilisables par les médias et reposant sur des formules accessibles et il a par ailleurs des effets d'entraînement sur les acteurs incités à l'utiliser. Quand il est régulier, il s'intègre dans une routine médiatique peu valorisée par les journalistes mais nécessaire et parfois rassurante. Au-delà du champ médiatique, le succès des palmarès est aussi le résultat d'une conjoncture politique spécifique, nous dit Sue Thomas en ouverture de son article sur le cas de l'Australie, conjoncture qui voit se télescoper le souci de la qualité des systèmes éducatifs, les discours alarmistes sur la crise de l'enseignement et le mauvais niveau des enseignants et les travaux de recherche analysant ces points. On le retrouve particulièrement dans ce numéro (et ailleurs) dans les cas de l'Angleterre, de l'Australie et des États-Unis. D'autres hypothèses peuvent être explorées comme la correspondance plus ou moins forte entre le palmarès et les différentes conceptions de leur métier mises en avant par les journalistes en éducation (Pons, 2014). 
Ces pistes ont toutes en commun de plaider pour une approche comparative, encore rare $^{5}$, permettant de prendre en compte une grande diversité de contextes. À cet égard, ce numéro aurait indéniablement mérité un meilleur équilibre entre pays développés et pays en développement. Nous l'avons ardemment visé sans pouvoir l'obtenir' ${ }^{6}$. Il nous paraîtrait également intéressant, pour ne pas avoir qu'une vision endogène du processus de médiatisation et de ses effets sur les problèmes éducatifs, de le comparer à d'autres formes de diffusion et de relais de la parole publique.

\section{BIBLIOGRAPHIE}

BALLAND L. (2009): Une sociologie politique de la crise de l'École: de la réussite d'un mythe aux pratiques enseignantes, thèse de doctorat, Université Paris-Ouest Nanterre.

FORESTIER Y. (2014): L'école, exception médiatique. La presse face aux enjeux des changements pédagogiques 1959-2008, thèse de doctorat, Université Paris - Sorbonne.

GERSTL-PEPIN C. I. (2007) : "Introduction to the Special Issue on the Media, Democracy, and the Politics of Education ", Peabody Journal of Education, n ${ }^{\circ}$ 82(1), p. 1-9.

GEWIRTZ S., DICKSON M., POWER S. (2004) : "Unravelling a "spun” policy: A case study of the constitutive role of "spin" in the education policy process ", Journal of Education Policy, $\mathrm{n}^{\circ}$ 19, p. 321-342.

HARLÉ M. (2004) : École et télévision: le choc des cultures. Réalité, mythe, imaginaire, Paris, l'Harmattan.

LINGARD B. \& RAWOLLE S. (2004) : « Mediatizing educational policy: The journalistic field, science policy, and cross-field effects », Journal of Educational Policy, 19, p. 361-380.

PONS X. (2014) : "Les journalistes français en éducation : une exploration typologique ", Éducation et sociétés, 33, 1, à paraître.

PONS X. (à paraître) : «La médiatisation de Pisa en France : travail journalistique et action publique", dans Julie Bouchard (dir.), La médiatisation de l'évaluation, à paraître.

VERGNIOUX A. (2013) : Grandes controverses en éducation, Berne, Peter Lang.

5. Voir la thèse pionnière de Mélusine Harlé (2004) qui compare le traitement médiatique de l'école dans les journaux télévisés français et américains dans les années 1990.

6. Néanmoins, il est probable qu'un tel équilibre n'aurait pas fondamentalement changé plusieurs des pistes, explications, conclusions fournies dans ce numéro, comme en témoignent les divers articles au numéro spécial de 2002 des Cahiers de la recherche sur l'éducation et les savoirs consacré à la médiatisation de l'école et des jeunes en Afrique. Dans divers pays de ce continent aussi, la médiatisation de l'école pose des questions politiques et idéologiques fondamentales. Dans ces systèmes scolaires aussi, les responsables politiques tentent de co-construire l'information médiatique légitime. Dans les journaux de ce continent aussi, on retrouve des logiques de présentation manichéennes et divers processus de réduction des enjeux etc. 